\title{
Broadband etching-free metal grating couplers embedded in titanium dioxide waveguides
}

\author{
MANON LAMY, Kamal HAMMANI, * JUAN AROCAS, ChRISTOPHE FINOT AND \\ JEAN-CLAude WEEBER
}

Laboratoire Interdisciplinaire Carnot de Bourgogne (ICB), UMR 6303 CNRS - Université Bourgogne Franche-Comté, 9 Av. A. Savary, BP 47870,
21078 Dijon Cedex, FRANCE

*Corresponding author: kamal.hammani@u-bourgogne.fr

Received XX Month XXXX; revised XX Month, XXXX; accepted XX Month XXXX; posted XX Month XXXX (Doc. ID XXXXX); published XX Month XXXX

Embedded metal grating couplers into titanium dioxide layer are proposed. A coupling efficiency better than $20 \%$ is experimentally demonstrated with a 3-dB bandwidth of $86 \mathrm{~nm}$ in agreement with simulation results. This allowed us to perform error-free transmissions of $10 \mathrm{Gbit} / \mathrm{s}$ wavelength multiplexed signals in the C-band. (C2017 Optical Society of America

OCIS codes: (130.0130) Integrated optics; (130.3130) Integrated optics materials; (050.2770) Gratings; (060.4510) Optical communications;

http://dx.doi.org/xx.xxx/OL.xx.xxxxxxx

The efficient coupling of light from an optical fiber to photonic waveguides remains an essential need for the emergence of integrated optical devices. The excitation of an integrated waveguide by an optical fiber is basically achieved by two ways known as end-fire coupling (or butt-coupling) or grating coupling. In the case of end-fire coupling, the optical fiber must be carefully aligned with respect to the (clean) waveguide input and sophisticated approaches such as cantilever adiabatic couplers [1] are needed to account for the large mismatch between the optical fiber and the waveguide mode size. On the other hand, grating couplers (GC) lead to very high coupling efficiencies [2] without the requirement of a highly accurate positioning of the optical fiber but at the cost of tedious fabrication processes. In particular, the etching depth of the grating couplers turns to be a critical parameter that can fully jeopardized their efficiency. To circumvent those limitations, couplers relying on metallic gratings have been recently proposed $[3,4]$. In those previous works, the grating couplers were deposited either on top of or beneath the photonic layer limiting the number of structural parameters available for the optimization of the couplers.

In this study, we investigate the situation of a metallic grating coupler embedded within the photonic guiding layer comprised of titanium dioxide deposited on a glass substrate. In particular, we optimize the location of the grating into the photonic waveguide and we show experimentally a factor two improvement of the coupling efficiency for the embedded grating as compared to the situation of the coupler deposited on top of the waveguide. Beyond their simple coupling efficiency characterizations, we also show that the embedded couplers are fully convenient for error-free transmission of $10 \mathrm{Gbit} / \mathrm{s}$ WDM signals.

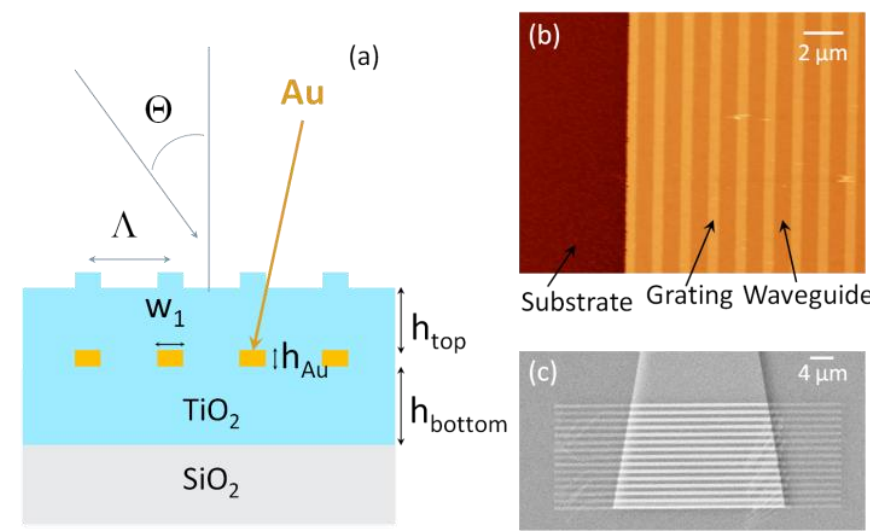

Fig. 1. (a) Embedded metal grating in titanium Dioxide layer (side view) (b) Atomic Force Microscope picture of the top surface. (c) SEM image of a fabricated device (top view)

A diagrammatic view of the embedded GCs we consider is depicted in Fig. 1(a). The GC is comprised of gold ridges (width $\mathrm{w}_{1}$, height $h_{A u}$ ) periodically distributed (period $\Lambda$ ) and embedded into a titanium dioxide $\left(\mathrm{TiO}_{2}\right)$ layer lying on a glass substrate. In our configuration, gold has been selected for its chemical stability to prevent alteration of the ridges during the encapsulation process. However, it is well-know that gold ridges can support localized surface plasmon resonances (LSPRs). The interaction of photonic modes with periodic arrangement of metallic nanostructures sustaining LSPRs has attracted much interest recently [5-7]. The spectral shaping of LSPRs and the generation of surface lattice resonances originating from the hybridization of the LSPR and the photonic modes have been demonstrated in this context. In the 
present case, the cross-sections of the gold ridges are such that they do not support LSPR in the telecom frequency range we consider. Hence, although of great interest, the contribution LSPR in metallic grating couplers is out of scope of the present study. Given that our GCs do not exploit the plasmonic properties of the metal, one can anticipate that cost effective CMOS compatible metals such as titanium nitride or aluminum could be conveniently used in place of gold. Figures 1(b) and 1(c) show an atomic force microscope image and a SEM image of the fabricated GC respectively. The GCs are sandwiched in between two layers of $\mathrm{TiO}_{2}$ with a total fixed height of $255 \mathrm{~nm}$ throughout the study. $\mathrm{TiO}_{2}$ has indeed been recognized [8-11] as a promising material for linear and non-linear integrated optics applications due to its high refractive index $(\mathrm{n}=2.45$ at $1.55 \mu \mathrm{m})$, broad transparency window and negligible two photon absorption in the telecom C-band. $\mathrm{TiO}_{2}$ can be readily deposited at low temperature making this material very convenient for the encapsulation of metallic GCs.

For a fixed thickness of the photonic waveguide, the period, the filling factor, the height and the distance $h_{\text {bottom }}$ [see Fig. 1(a)] have to be considered for an optimum design. As discussed in more details in the following, the encapsulation of the GC is obtained by a conformal deposition of a $\mathrm{TiO}_{2}$ layer onto the metal grating in such a way that a replica of the metal grating is created at the top surface of the upper $\mathrm{TiO}_{2}$ layer. We carried out an optimization of the structural parameters of two-dimensional GCs by using a commercial finite-element software. Assuming a Gaussian beam (free-space wavelength $1550 \mathrm{~nm}$ ) falling at an angle of incidence of $\Theta=30^{\circ}$ (corresponding to our experimental configuration), the best set of parameters (giving the maximum coupling efficiencies) for a fixed total thickness of $255 \mathrm{~nm}$ have been obtained thanks to Monte-Carlo routine and are summarized in Table1.

Table 1. Best set of parameters and coupling efficiency (CE) depending on the configuration and on the initial polarized Gaussian beam

\begin{tabular}{|c|c|c|c|}
\hline & & TE & TM \\
\hline \multirow{5}{*}{$\begin{array}{l}\text { Embedded } \\
\text { configuration } \\
\text { (see sketch } \\
\text { Fig 1a) }\end{array}$} & $\Lambda$ & $1235 \mathrm{~nm}$ & $1396 \mathrm{~nm}$ \\
\hline & $\mathrm{W}_{1}$ & $386 \mathrm{~nm}$ & $666 \mathrm{~nm}$ \\
\hline & $\mathrm{h}_{\mathrm{Au}}$ & $14 \mathrm{~nm}$ & $56 \mathrm{~nm}$ \\
\hline & $\mathrm{h}_{\text {bottom }}$ & $63 \mathrm{~nm}$ & $65 \mathrm{~nm}$ \\
\hline & CE & $\begin{array}{c}\sim 30 \% \\
(\sim-5 \mathrm{~dB})\end{array}$ & $\begin{array}{c}\sim 50 \% \\
(\sim-3 \mathrm{~dB})\end{array}$ \\
\hline \multirow{4}{*}{$\begin{array}{c}\text { Top } \\
\text { configuration } \\
\text { (see sketch } \\
\text { Fig 5a) }\end{array}$} & $\Lambda$ & $1177 \mathrm{~nm}$ & $1421 \mathrm{~nm}$ \\
\hline & $\mathrm{w}_{1}$ & $502 \mathrm{~nm}$ & $165 \mathrm{~nm}$ \\
\hline & $\mathrm{h}_{\mathrm{Au}}$ & $59 \mathrm{~nm}$ & $56 \mathrm{~nm}$ \\
\hline & $\mathrm{CE}$ & $\begin{array}{c}\sim 20 \% \\
(\sim-7 \mathrm{~dB})\end{array}$ & $\begin{array}{c}\sim 15 \% \\
(\sim-8 \mathrm{~dB})\end{array}$ \\
\hline
\end{tabular}

The embedded configuration allows to reach higher coupling efficiencies for both TE and TM polarization compared to the top configuration. Moreover, while the difference between TE and TM polarization in top configuration is not significant, the TM polarization is clearly more efficient than the TE one with the embedded configuration. Therefore, in the following, we focus our attention on the TM polarization.

Figure 2 provides an overview of the impact of each parameter on the coupling efficiency per facet. When the period increases, as expected the maximum efficiency is shifted toward longer wavelength [Fig. 2(a)] and the highest coupling is obtained for filling factors close to $50 \%$ [Fig. 2(b)]. Regarding the impact of the position of the embedded grating, it is clear from Fig. 2(c) that the coupling efficiency is higher when the grating is not directly on the substrate [3]. Actually, the depth of embedded grating is related to the mode field distribution of the unperturbed waveguide. More precisely, the grating has to be positioned where the power flow (i.e. the Poynting vector) is the highest into the layer. Another variable parameter is the height of the grating which is not critical for the coupling efficiency beyond a minimal thickness. However, when the height of the grating increases, the optimal wavelength is shifted towards higher wavelength [Fig. 2(d)] due to an increase of the effective index. Moreover, beyond a certain value (here $56 \mathrm{~nm}$ ), the height of the grating becomes deleterious and leads to lower efficiency.
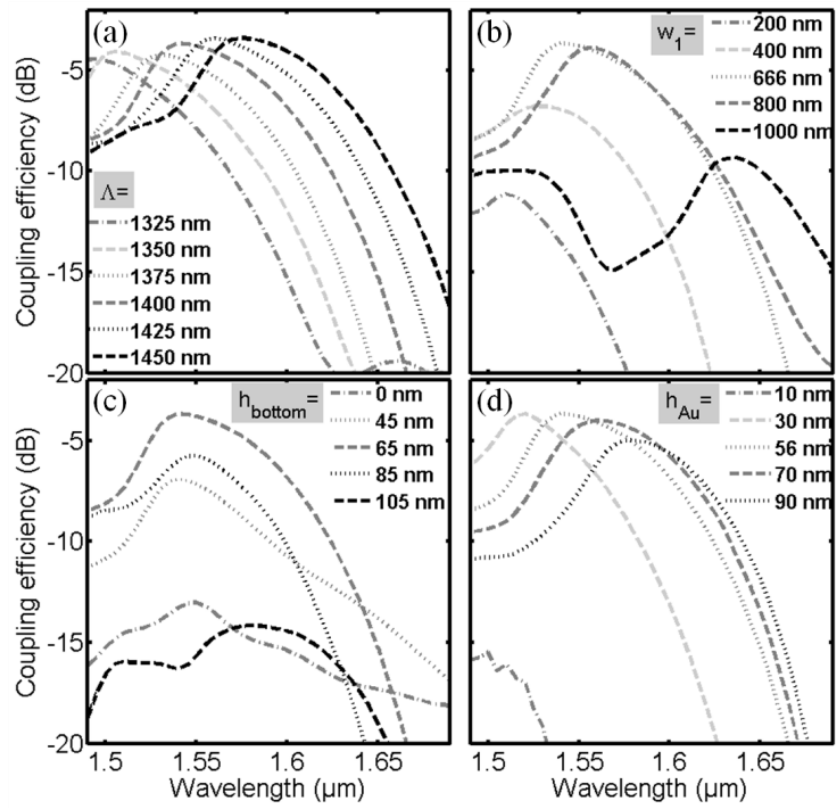

Fig. 2. Coupling efficiency as a function of (a) the period, (b) the filling factor, (c) the position and (d) the thickness of the metal embedded grating. For non-varying parameters, the values are $\Lambda=1400 \mathrm{~nm}$, $w_{1}=666 \mathrm{~nm}, h_{\mathrm{Au}}=56 \mathrm{~nm}, h_{\text {bottom }}=65 \mathrm{~nm}$ and $h_{\text {top }}=255 \mathrm{~nm}-h_{\text {bottom }}$.

Based on those results, embedded GCs have been obtained using the following process flow. First, a $\mathrm{TiO}_{2}$ layer (thickness $69 \mathrm{~nm}$ ) is deposited on a glass substrate by DC magnetron sputtering of a $99.9 \%$ pure titanium target. Next, the gold gratings are fabricated on top of the $\mathrm{TiO}_{2}$ layer by standard electron-beam lithography, thermal metal evaporation (3nm of chromium used as an adhesion layer $+56 \mathrm{~nm}$ of gold) followed by a lift-off process. Then the metal gratings are encapsulated by depositing a second $\mathrm{TiO}_{2}$ layer (thickness 193nm). An overlay electron lithography using alignment marks and eventually a dry-etching of the $\mathrm{TiO}_{2}$ layers are conducted to fabricate the photonic structures (waveguides and tapers). We have fabricated several devices with the period varying from $1300 \mathrm{~nm}$ to $1500 \mathrm{~nm}$ by step of $25 \mathrm{~nm}$. Finally, the width of the grating is about $610 \mathrm{~nm}$ (targeted 666 $\mathrm{nm}$ ). Note that two coupling tapers, where the gratings are embedded, are used to achieve beam spot conversion into a 1.5 $\mu \mathrm{m}$-wide rib waveguide. The total length of the device including these $85-\mu \mathrm{m}$ long tapers is $575 \mu \mathrm{m}$. 
The experimental setup is sketched in Fig. 3(a). Optical fibers equipped with focusers are used to couple light in and out the waveguide. The focusers generate a spot with a diameter around $10 \mu \mathrm{m}$ at a working distance of $8 \mathrm{~mm}$ and are tilted at an angle of $30^{\circ}$ with respect to the normal to the substrate. An IR and a visible camera mounted on a vertically oriented microscope are operated simultaneously to align accurately the focuser onto the GCs (see Figs 3(b) and 3(c) respectively). The light source we use is generated from a high peak power picosecond laser, centered at $1550 \mathrm{~nm}$ injected into a highly nonlinear fiber with anomalous dispersion. This apparatus generates a supercontinuum [12] with a spectrum ranging from $1300 \mathrm{~nm}$ to $1900 \mathrm{~nm}$. The polarization of the light injected into the input focuser is adjusted by polarization controllers and is oriented to be TM. At the distal end of the waveguide, the transmitted light decoupled by the grating is collected by the output focuser and analyzed with an optical spectral analyzer (OSA) or an accurate powermeter (PM).

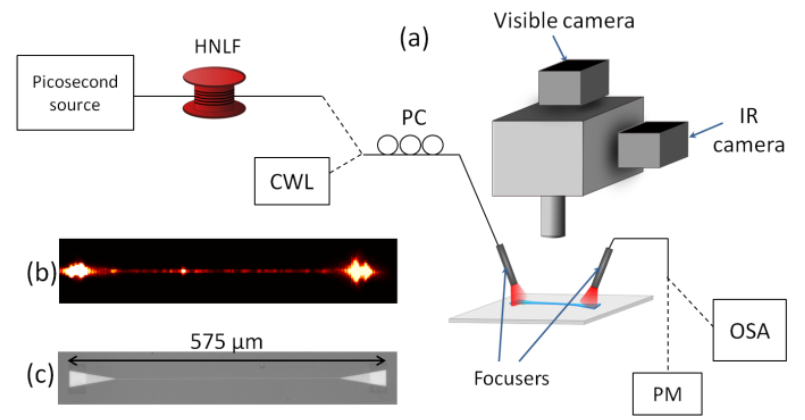

Fig. 3. (a) Experimental Setup. PC: polarization controller, OSA: optical spectrum analyzer, PM: powermeter, CWL: continuous wave laser, HNLF: highly nonlinear fiber. Top view of under test $1.5 \mu \mathrm{m}$-wide waveguide with (b) IR or (c) visible camera.

For the quantitative measurement of the coupling efficiencies, a thick gold layer on a glass substrate is used as a highly reflecting surface. The light collected by the output focuser after specular reflection of the incident light onto the gold mirror is optimized and a reference spectrum is recorded. By normalizing the transmission spectra along the waveguide to the reference spectrum, the spectral dispersion of the coupling efficiency for each grating coupler is readily obtained. Taking into account the symmetry of the coupling in and the coupling out configuration in our setup and neglecting the confinement and material losses experienced upon propagation, the coupling efficiency per facet is defined as half the transmission loss along the entire waveguide.

Figure 4(a) displays the coupling efficiency per facet for GCs with period around $1400 \mathrm{~nm}$ (optimal period in simulations). As expected from Fig. 2(a), we observe a red shift of the optimal wavelength with the increase of the GC period. Figure 4(b) compares the numerical and experimental results obtained for the optimal experimental period $\Lambda=1375 \mathrm{~nm}$. The dashed curve shows the optimized coupling efficiency that can be obtained after a full optimization of the GC parameters. The dotted grey curve is obtained by using for the numerical modeling the actual parameters ( $\mathrm{h}_{\text {bottom }}=69 \mathrm{~nm}, \mathrm{~h}_{\text {top }}=193 \mathrm{~nm}, \mathrm{w}=610 \mathrm{~nm}, \mathrm{~h}_{\mathrm{Au}}=56 \mathrm{~nm}$ ) measured after fabrication of the GC whereas the solid line displays the experimental coupling efficiencies obtained for this GC. The experimental coupling efficiency per facet features a maximum at $1550 \mathrm{~nm}$ around $-7 \mathrm{~dB}(20 \%)$ and a $3 \mathrm{~dB}$-bandwidth of $86 \mathrm{~nm}(1 \mathrm{~dB}$ bandwidth $=50 \mathrm{~nm})$ in fairly good agreement with the numerical results obtained with the actual parameters of the
GC. We note however a decrease of about $2 \mathrm{~dB}$ between the experimental and the numerical results (black dashed curve) attributed to the propagation losses along the waveguide and mainly related to fabrication defects [see scattering spots in Fig. 3(b)]. In addition, by referring to the optimum performances of the GC, we note that an improvement by about $2 \mathrm{~dB}$ of the coupling efficiency per facet is expected by a further optimization of the GC parameters. This waveguide is polarization-sensitive, with a measured extinction ratio of $12.9 \mathrm{~dB}$.
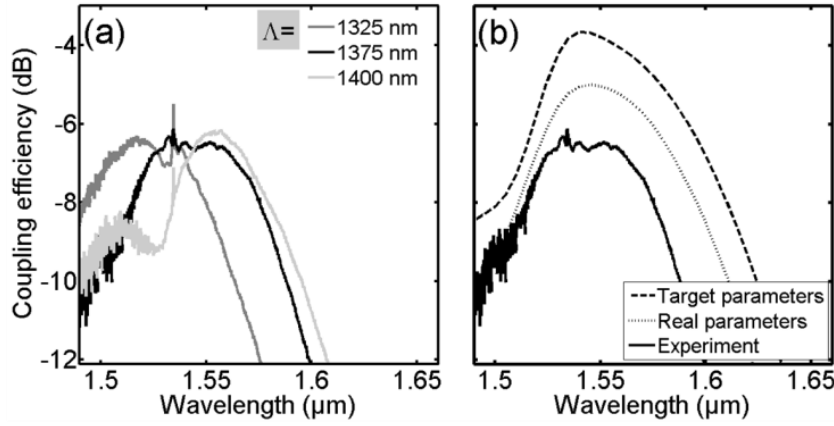

Fig. 4. Coupling efficiency per facet of embedded grating coupler as a function of the injected wavelength (a) for several period. (b).The best experimental results $(\Lambda=1375 \mathrm{~nm}$, solid black curve $)$ are compared with numerical simulations taking into account real parameters (dotted grey curve) and optimum parameters (dashed black curve).

To go further, we have fabricated GCs deposited on top of the photonic waveguides [Fig. 5(a)] at the same time (with the same lithography design). Thus the period and the filling factor of the GC are identical as in the case of the embedded gratings. Therefore, the TM mode is not optimally coupled due to a wrong filling factor. More precisely, while the optimal coupling (see Table 1) is expected to be $-8 \mathrm{~dB}$, with our set of experimental parameters, the coupling efficiency should drop down to $-13 \mathrm{~dB}$ per facet. In our experiment, we actually measured a total loss close to $-30 \mathrm{~dB}$ which means a coupling efficiency being about $-15 \mathrm{~dB}$ per facet, validating qualitatively the predictions of the numerical simulations. Regarding the TE mode, the same grating with a filling factor close to $50 \%$ can be optimal but of course not at $1550 \mathrm{~nm}$. Indeed, the effective index is significantly higher and leads to a shift towards higher wavelength. Thus by operating the supercontinuum source, the spectra displayed in Fig. 5(b) were obtained. The best result was about $-10 \mathrm{~dB}$ (where the simulation gives $-7 \mathrm{~dB}$ ) which is still lower than the efficiency with the embedded grating coupler (expected to be $-5 \mathrm{~dB}$ ). This demonstrates the potential of such embedded structures to couple light in photonic waveguides and confirms that the embedded GCs are a particularly powerful way to couple the TM-polarized light.

The superior performances of embedded GCs as compared to deposited GCs can be understood qualitatively from the fact that the grating is located at the maximum of the power density of the unperturbed waveguide but also from the interaction of the incident light with the grating located at the upper surface of the photonic waveguide. The role of this upper grating can be evaluated on the basis of numerical modeling. Indeed, for the optimal GC we have checked that the coupling efficiency of the embedded coupler is reduced by a factor 2 if the top grating is removed. The interaction of the top grating and embedded grating is thus a key ingredient for the optimum performance of the embedded GCs. The intrinsic properties of the embedded GCs have been discussed so far but it is also worthy to demonstrate those 
GCs in the context of practical applications such as a high-bit rate traffic.
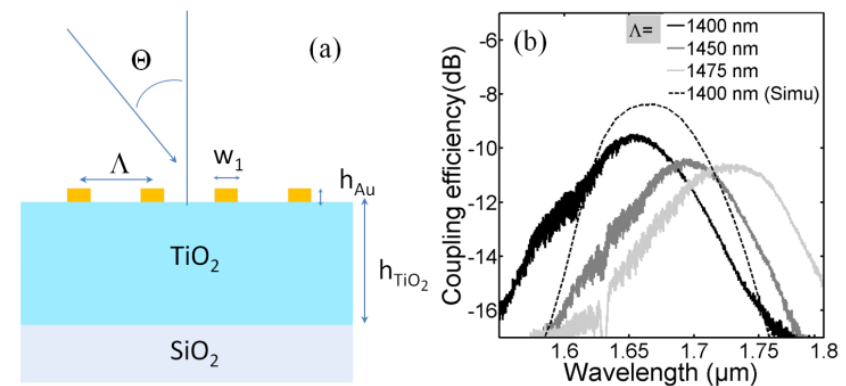

Fig. 5. (a) Top metal grating on titanium dioxide (side view). (b) Coupling efficiency per facet of embedded grating coupler as a function of the injected wavelength for several periods.

To demonstrate the efficiency of the embedded coupler device, we have transmitted a $10 \mathrm{Gbit} / \mathrm{s}$ signal at standard telecommunications wavelengths. More precisely, we used enhanced small form-factor pluggable (SFP+) to transmit and receive light. Those transceivers are commonly used as part of wavelength-division multiplexing optical networks. The $10 \mathrm{Gbit} / \mathrm{s}$ signal is coded through a $2^{31}$ pseudo random bit sequence (PRBS) generator. At the output, a bit error rate tester (BERT) and a high speed sampling oscilloscope are used to evaluate the bit error rate (BER) and monitor the eye diagram. For SFP+ typical of coarse wavelength multiplexing emitting at 1530, 1550 and $1570 \mathrm{~nm}$, BER lower than $10^{-12}$ are achieved, indicating error-free transmission. For SFP+ centered at $1510 \mathrm{~nm}$, the BER decreases to $1.610^{-11}$ due to $2 \mathrm{~dB}$ extra-loss that prevents the integrated amplifier and reshaper to perfectly work.
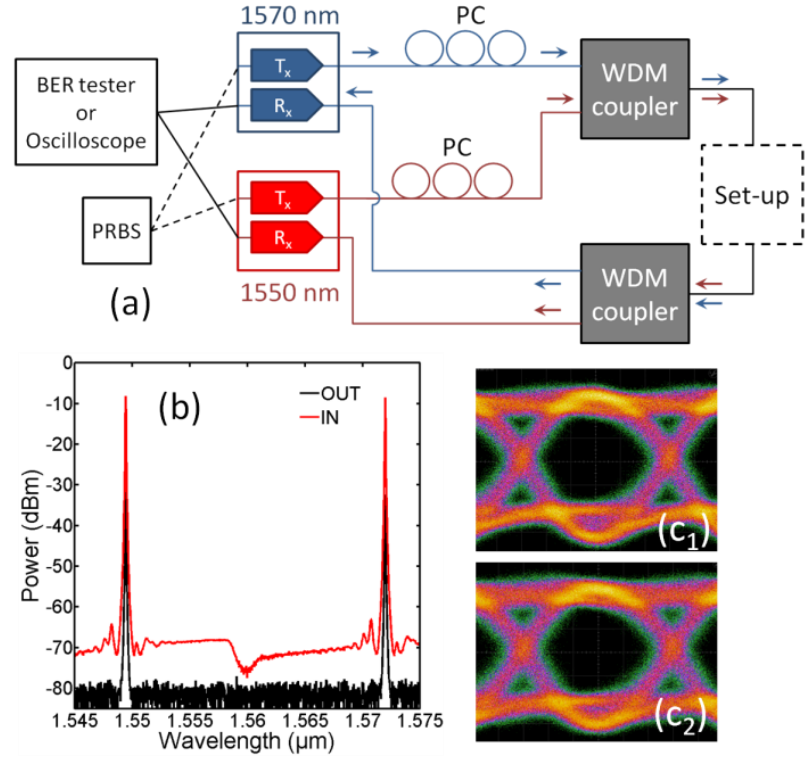

Fig. 6 (a) Example of experimental setup for $10 \mathrm{Gbit} / \mathrm{s}$ transmission. Each SFP+ is composed of a transmitter Tx and a receiver Rx. WDM = wavelength division multiplexer. The square called 'set-up' corresponds to the setup shown in Fig. 3(a). (b) Optical spectrum in copropagation configuration (c) Eye diagrams at $1550 \mathrm{~nm}$ (c1) and 1570 $\mathrm{nm}$ (c2) in counter-propagation configuration.

To go beyond, two WDM experiments have been performed. First, a co-propagating configuration [Fig. 6(a)] has been considered with a signal centered at $1550 \mathrm{~nm}$ and another centered at $1570 \mathrm{~nm}$ as shown by the spectrum Fig. 6(b). At 1550 $\mathrm{nm}$, the transmission is error-free whereas at $1570 \mathrm{~nm}$ the BER is about $410^{-8}$. Second, previous signals have been injected in a counter-propagation configuration. As demonstrated by the eye diagrams [Fig. 6(c)], the transmission is excellent with clear open eye. More precisely, at $1550 \mathrm{~nm}$, the transmission is still error-free whereas at $1570 \mathrm{~nm}$ the BER is about $7.5 .10^{-7}$. The degradation between single and multi channel configurations is explained by the insertion of two optical wavelength multiplexers leading to $2 \mathrm{~dB}$ of extra-loss.

To conclude, we have shown, for the first time to our knowledge, the fabrication of metal grating couplers in gold embedded into a titanium dioxide waveguide. We demonstrate coupling efficiency greater than $20 \%$ at $1550 \mathrm{~nm}$ for a TM polarization. The bandwidth of this device is about $86 \mathrm{~nm}$ at $3 \mathrm{~dB}$. The comparison with a metal grating coupler on top of the waveguide confirms the higher potential of the embedded device. Our simulations are in good agreement with our numerical results even though small discrepancies can be explained by fabrication defects. However, simulations demonstrated that efficiencies close to $45 \%$ could be reached. The device has been tested in real configuration using integrated transceivers (SFP+) to transmit coded signal at $10 \mathrm{Gbit} / \mathrm{s}$ without any error. To increase the performances of such device, several ways should be explored with in particular a metallic pad on the substrate that acts as a mirror or the optimization of coupler shape by using parabolic grating [4]. Such coupling efficiency will allow to consider the titanium dioxide platform as a serious candidate for nonlinear applications in near infrared [9].

Funding. PARI PHOTCOM Région Bourgogne, Labex Action (ANR11-LABX-01-01) and FEDER-FSE Bourgogne 2014/2020.

Acknowledgment. We thank J. Fatome and M. G. Nielsen for advices and fruitful discussion on experimental and numerical parts.

\section{References}

1. L. Chen, C. R. Doerr, Y. K. Chen, and T. Y. Liow, IEEE Photonic Tech. L. 22, 1744 1746 (2010).

2. D. Taillaert, P. Bienstman, and R. Baets, Opt. Lett. 29, 2749-2751 (2004).

3. S. Scheerlinck, J. Schrauwen, F. Van Laere, D. Taillaert, D. Van Thourhout, and R. Baets, Opt. Express 15, 9625-9630 (2007).

4. L. Wang, Y. Wang, and X. Zhang, Optics express 20, 17509-17521 (2012).

5. S. R. K. Rodriguez, Y. T. Chen, T. P. Steinbusch, M. A. Verschuuren, A. F. Koenderink, and J. Gómez Rivas, PHYSICAL REVIEW B 90 (2014).

6. M. Fevrier, P. Gogol, J. M. Lourtioz, and B. Dagens, Optics Express 21, 24504 24513 (2013).

7. A. Christ, S. G. Tikhodeev, N. A. Gippius, J. Kuhl, and H. Giessen, Physical Review Letters 91, 183901-183901 (2003).

8. J. D. B. Bradley, C. C. Evans, J. T. Choy, O. Reshef, B. Parag, F. Parsy, K. C. Phillips, M. Lon, and E. Mazur, Optics express 20, 8336-8346 (2012).

9. C. C. Evans, K. Shtyrkova, J. D. B. Bradley, O. Reshef, E. Ippen, and E. Mazur, Opt. Express 21, 18582-18591 (2013).

10. O. Reshef, K. Shtyrkova, M. G. Moebius, S. Griesse-Nascimento, S. Spector, C. C. Evans, E. Ippen, and E. Mazur, Journal of the Optical Society of America B 32, 2288-2293 (2015).

11. C. C. Evans, C. Liu, and J. Suntivich, Opt. Express 23, 11160-11169 (2015).

12. J. M. Dudley, G. Genty, and S. Coen, Revi. Mod. Phys. 78, 1135-1184 (2006). 